\title{
Natural solution in the refined Gribov-Zwanziger theory
}

\author{
D.J. Thelan \& J.A. Gracey, \\ Theoretical Physics Division, \\ Department of Mathematical Sciences, \\ University of Liverpool, \\ P.O. Box 147, \\ Liverpool, \\ L69 3BX, \\ United Kingdom.
}

\begin{abstract}
We analyse the one loop effective action of the Gribov-Zwanziger Lagrangian and use the local composite operator formalism to include the most general Becchi-Rouet-StoraTyutin (BRST) dimension two mass operator for the localizing ghost fields. We show that the energetically favourable colour channel corresponds to what is known as the $\mathcal{R}$ direction.
\end{abstract}


In recent years there has been interest in understanding the infrared behaviour of the gluon and Faddeev-Popov ghost propagators in Quantum Chromodynamics (QCD). This is motivated in the main by their relation to confinement. Gluons, which are the quanta mediating the strong nuclear force, are not observed in nature unlike the other vector bosons in the full standard model. In this respect the gluon propagator does not have the canonical behaviour associated with an observed fundamental particle which is the presence of a simple pole at the mass shell value. While its high energy asymptotic properties are similar to those of say a photon, in the low energy regime a pole at zero momentum does not apparently emerge. Evidence for this is provided by several approaches. These are lattice gauge theory, Schwinger-Dyson methods and the Hamiltonian approach (in the Coulomb gauge), 1]. The majority activity in recent years has primarily centred on the Landau gauge. Though in this gauge, which we concentrate on here, there are several scenarios. In one case at zero momentum the gluon propagator freezes to a non-zero finite value without any singular behaviour. A selection of articles demonstrating this are, for example, [2, 3, 4, 5, 6, 7, 8, 9, 10, 11]. However, in analysing non-abelian gauge theories at low energies one has to be aware of global considerations. In the case of Landau gauge fixed QCD the main problem one has to be aware of is the Gribov problem, [12. Stated briefly it is not possible to fix the gauge globally as there are different gauge copies satisfying the same gauge fixing condition which have to be factored out of the path integral to ensure that there is no double counting. Although there are additional subtle issues as to whether accounting for such Gribov copies produces a unique gauge configuration, Gribov demonstrated, [12, that their presence affected the properties of the gluon and Faddeev-Popov propagators at low momenta. The former is suppressed and freezes to a zero value while the latter enhances with a double pole structure in $p^{2}$ where $p$ is the momentum. Clearly this is not the behaviour found in recent years from lattice and some Schwinger-Dyson solutions, 2, 3, 4, 5, 6, 7, 8, 9, 10, 11, On terminology the original Gribov scenario of [12] is referred to as the scaling solution and the non-zero freezing is termed the decoupling solution, [1]. Indeed recently various lattice analyses have shed some light as to why the latter emerges in the data as opposed to the scaling case, [13, 14]. However, Schwinger-Dyson solutions do find the scaling solution too. Moreover, the Coulomb gauge Hamiltonian approach strongly supports the Gribov confinement picture and connects the dual Meissner effect with the Gribov-Zwanziger confinement analysis, 1, 15, 16, 17.

After Gribov's definitive analysis of the gauge fixing problem in the Landau gauge, [12, the non-local resultant Lagrangian was localized in a series of articles, [18, 19, 20, 21, 22, 23, 24, 25, 26]. This produced a local renormalizable Lagrangian which meant that one could carry out loop computations. For instance, the two loop gap equation satisfied by the Gribov mass $\gamma$, which derives from the path integral cutoff, was determined in [12, 25, 27]. Also the zero momentum freezing of the gluon propagator and the Faddeev-Popov ghost enhancement were confirmed at one and two loops respectively. However, the localization process of [18, 19, 20, 21, 22, 23, 24, 25, [26] which Zwanziger constructed required two sets of extra localizing spin-1 ghost fields. One set is bosonic and the other is Grassmann. These play a passive role at high energy but affect the low momentum behaviour of the propagators. Indeed they have interesting dynamics in themselves. For instance, it was shown in 28] that the adjoint projection of the bosonic localizing ghost had an enhanced behaviour in its longitudinal part. However, like the original Gribov formulation, this pure Gribov-Zwanziger Lagrangian does not produce the decoupling properties observed in current data. To model this the Gribov-Zwanziger Lagrangian was refined in [29] to include a mass operator for the localizing ghost fields. Such a mass term alters the infrared properties of their propagators as well as that of the gluon. In particular the gluon has a non-zero value at zero momentum. The analysis was based on the local composite operator (LCO) formalism, [30, 31, 32], and constructed the effective potential satisfied by the mass operator. It was then shown that the potential had a minimum at a non-zero value indicating that the condensation 
of the operator would produce a mass term for the localizing ghosts thereby modelling the non-zero frozen gluon propagator behaviour, 29]. However, the analysis of [29] did not use the most general possible localizing ghost mass operator. In [33] the most general dimension two BRST invariant operator was considered based on all the possible colour tensors. One feature was that the frozen gluon propagator behaviour did not have a unique solution from the LCO mechanism. Indeed it was shown in [33] that other colour structures not considered in [29] could reproduce lattice data. Therefore to reconcile whether there is a preferred colour tensor structure we will extend here the analysis of [29] to the general case of [33] and complete the programme begun in [33. The aim is to see if there is indeed a unique minimum solution to the corresponding LCO effective potential for the general operator. If there is a unique minimum we can therefore regard this as the energetically favourable operator condensation colour direction. Moreover, once determined the properties of the corresponding propagators at low momentum can therefore provide potential tests on future data to ascertain whether this is the underlying Lagrangian structure.

To set the background to the problem we recall Gribov's observation, [12], that the QCD action has to be modified to account for the effect of copies deriving from the global gauge fixing ambiguity. The subsequent non-local Gribov Lagrangian which depends on the Gribov mass is, [12, 25,

$$
L^{\text {Gribov }}=L^{\mathrm{QCD}}+\frac{\gamma^{4}}{2} f^{e a c} f^{e b d} A_{\mu}^{a}\left(\frac{1}{\partial^{\nu} D_{\nu}}\right)^{c d} A^{b \mu}-\frac{d N_{A} \gamma^{4}}{2 g^{2}}
$$

where $A_{\mu}^{a}$ is the gluon, $N_{A}$ is the adjoint representation dimension with $1 \leq a \leq N_{A}$, $d$ is the spacetime dimension and

$$
L^{\mathrm{QCD}}=-\frac{1}{4} G_{\mu \nu}^{a} G^{a \mu \nu}-\frac{1}{2 \alpha}\left(\partial^{\mu} A_{\mu}^{a}\right)^{2}-\bar{c}^{a} \partial^{\mu} D_{\mu} c^{a}+i \bar{\psi}^{i I} \not D \psi^{i I}
$$

is the QCD Lagrangian for $N_{f}$ massless quarks $\psi^{i I}$ with $1 \leq i \leq N_{f}$ and $1 \leq I \leq N_{F}$ where $N_{F}$ is the fundamental representation dimension and $c^{a}$ is the Faddeev-Popov ghost. The presence of $\gamma$ results from imposing the no pole condition, [12, which equates to defining the Gribov horizon in configuration space. From (11) this is, [12, 25],

$$
f^{e a c} f^{e b d}\left\langle A_{\mu}^{a}\left(\frac{1}{\partial^{\nu} D_{\nu}}\right)^{c d} A^{b \mu}\right\rangle=\frac{d N_{A}}{g^{2}} .
$$

Given that the gluon propagator depends on $\gamma$ this condition constrains $\gamma$ to satisfy a gap equation. In other words, [12, 20, 25], only when the gap equation is imposed is one in the gauge theory. To circumvent the inability to perform computations with a Lagrangian with a non-local term, in a series of articles, [18, 19, 20, 21, 22, 23, 24, 25, 26, Zwanziger localized the horizon term of (1) with the introduction of localizing ghost fields. In the current formulation of this these fields are $\xi_{\mu}^{a b}, \rho_{\nu}^{a b}, \omega_{\mu}^{a b}$ and $\bar{\omega}_{\mu}^{a b}$ where the first two are bosonic and the other two are Grassmann. The latter play the same role to the bosonic localizing ghosts as the Faddeev-Popov ghosts do for the gauge field. The full localized Lagrangian is, 25,

$$
\begin{aligned}
L^{\mathrm{GZ}}= & L^{\mathrm{QCD}}+\frac{1}{2} \rho^{a b \mu} \partial^{\nu}\left(D_{\nu} \rho_{\mu}\right)^{a b}+\frac{i}{2} \rho^{a b \mu} \partial^{\nu}\left(D_{\nu} \xi_{\mu}\right)^{a b}-\frac{i}{2} \xi^{a b \mu} \partial^{\nu}\left(D_{\nu} \rho_{\mu}\right)^{a b} \\
& +\frac{1}{2} \xi^{a b \mu} \partial^{\nu}\left(D_{\nu} \xi_{\mu}\right)^{a b}-\bar{\omega}^{a b \mu} \partial^{\nu}\left(D_{\nu} \omega_{\mu}\right)^{a b}-\frac{1}{\sqrt{2}} g f^{a b c} \partial^{\nu} \bar{\omega}_{\mu}^{a e}\left(D_{\nu} c\right)^{b} \rho^{e c \mu} \\
& -\frac{i}{\sqrt{2}} g f^{a b c} \partial^{\nu} \bar{\omega}_{\mu}^{a e}\left(D_{\nu} c\right)^{b} \xi^{e c \mu}-i \gamma^{2} f^{a b c} A^{a \mu} \xi_{\mu}^{b c}-\frac{d N_{A} \gamma^{4}}{2 g^{2}} .
\end{aligned}
$$

Its properties are well established, [25, 26, 27]. For the purposes of this article the key ones are that the Faddeev-Popov ghosts as well as $\omega_{\mu}^{a b}$ enhance in the infrared when the gap equation 
for $\gamma$ is satisfied. Hence the Kugo-Ojima confinement criterion, 34, is fulfilled. Indeed more recently an analysis of this condition for other gauges such as Coulomb and the maximal abelian gauge has been provided in 35]. There the Kugo-Ojima confinement condition was generalized to these additional gauges and criteria were given to differentiate between the Higgs or Coulomb phases of BRST symmetric gauge theories and the colour confining phase. Indeed this represents progress towards having a universal criterion for colour confinement. In addition there is an infrared enhancement for the bosonic localizing ghosts in (4). More specifically it has been shown in 28] that the adjoint projection of the longitudinal part of the $\xi_{\mu}^{a b}$ propagator enhances in the infrared. It was argued that this feature reflected the Goldstone boson associated with the spontaneous breaking of a BRST related symmetry of the Lagrangian in the presence of a constraint. The final property of (11) which is relevant is that the gluon propagator is suppressed in the infrared. The last property can be seen in the propagators of (4) which are

$$
\begin{aligned}
\left\langle A_{\mu}^{a}(p) A_{\nu}^{b}(-p)\right\rangle & =-\frac{\delta^{a b} p^{2}}{\left[\left(p^{2}\right)^{2}+C_{A} \gamma^{4}\right]} P_{\mu \nu}(p),\left\langle A_{\mu}^{a}(p) \xi_{\nu}^{b c}(-p)\right\rangle=\frac{i f^{a b c} \gamma^{2}}{\left[\left(p^{2}\right)^{2}+C_{A} \gamma^{4}\right]} P_{\mu \nu}(p) \\
\left\langle\xi_{\mu}^{a b}(p) \xi_{\nu}^{c d}(-p)\right\rangle & =-\frac{\delta^{a c} \delta^{b d}}{p^{2}} \eta_{\mu \nu}+\frac{f^{a b e} f^{c d e} \gamma^{4}}{p^{2}\left[\left(p^{2}\right)^{2}+C_{A} \gamma^{4}\right]} P_{\mu \nu}(p),\left\langle A_{\mu}^{a}(p) \rho_{\nu}^{b c}(-p)\right\rangle=0 \\
\left\langle\rho_{\mu}^{a b}(p) \rho_{\nu}^{c d}(-p)\right\rangle & =\left\langle\omega_{\mu}^{a b}(p) \bar{\omega}_{\nu}^{c d}(-p)\right\rangle=-\frac{\delta^{a c} \delta^{b d}}{p^{2}} \eta_{\mu \nu},\left\langle\xi_{\mu}^{a b}(p) \rho_{\nu}^{c d}(-p)\right\rangle=0
\end{aligned}
$$

where $P_{\mu \nu}(p)=\eta_{\mu \nu}-p_{\mu} p_{\nu} / p^{2}$. The gluon suppression has been checked at one loop, 36], which is due in the main to the fact that (44) is renormalizable, [25, 37, 38], allowing one to perform loop computations.

In summarizing these general features of the Gribov construction which persist in loop calculations, it is evident that they are not observed on the lattice, [2, 3, 4, 15, 6, 7, 8, 9, 10, 11. Instead the numerical data indicates that the gluon propagator freezes to a non-zero value and the Faddeev-Popov ghost is not enhanced in the infrared. This decoupling behaviour has been observed in one set of Schwinger-Dyson solutions, [11. However, in [29] a BRST invariant mass operator for the localizing ghosts was introduced and its effect on the structure of the theory in the infrared was analysed. Briefly a frozen gluon propagator and non-enhanced Faddeev-Popov ghost propagator emerged in the quantum analysis which mimics the lattice observations. It was subsequently pointed out in 33 . that the particular choice of BRST invariant mass operator of 29] was not unique to modelling the decoupling solution. Indeed it was not the most general possible BRST dimension two operator from a group theoretic point of view. While one could in principle add a mass operator for the localizing ghosts to (44) such a term would have no origin in the original Gribov Lagrangian, (11). Therefore, a mass term for the extra fields can be established via a non-zero vacuum expectation value for the mass operator. In other words if one computed the effective potential for the mass operator and found that it was a minimum at a non-zero value which was energetically more favourable than the massless localizing ghost case then the mass operator would condense. Thus the non-zero vacuum expectation value would produce the necessary masses to model a frozen gluon propagator and non-enhanced FaddeevPopov ghost propagator. In [29] such an analysis was performed for the operator considered there. However, as noted in 33 by restricting the seed operator to a specific colour direction in colour space it was not clear whether the vacuum solution which emerged was the energetically most favourable one. Therefore, we provide the analysis for the most general BRST dimension two localizing ghost operator and aim to establish the direction in colour space which is the energetically favourable.

The most general BRST dimension two operator was introduced in [33] and in the same 
(non-orthogonal) basis is

$$
\mathcal{O}=\left[\mu_{\mathcal{Q}}^{2} \delta^{a c} \delta^{b d}+\mu_{\mathcal{W}}^{2} f^{a c e} f^{b d e}+\frac{\mu_{\mathcal{R}}^{2}}{C_{A}} f^{a b e} f^{c d e}+\mu_{\mathcal{S}}^{2} d_{A}^{a b c d}+\frac{\mu_{\mathcal{P}}^{2}}{N_{A}} \delta^{a b} \delta^{c d}+\mu_{\mathcal{T}}^{2} \delta^{a d} \delta^{b c}\right] \mathcal{O}^{a b c d}
$$

where the colour directions are each associated with a mass parameter, $\mu_{\mathcal{I}}^{2}$. We will use the label $\mathcal{I}$ to indicate the various possible colour channels defined by the colour tensor. For reference in [29] channel $\mathcal{Q}$ was the main focus. In (6) $f^{a b c}$ are the structure function of the colour group and, 39],

$$
d_{A}^{a b c d}=\frac{1}{6} \operatorname{Tr}\left(T_{A}^{a} T_{A}^{(b} T_{A}^{c} T_{A}^{d)}\right)
$$

which is totally symmetric in its colour indices. The field content of the operator is, 33],

$$
\mathcal{O}^{a b c d}=\frac{1}{2}\left[\rho^{a b} \rho^{c d}+i \xi^{a b} \rho^{c d}-i \rho^{a b} \xi^{c d}+\xi^{a b} \xi^{c d}\right]-\bar{\omega}^{a b} \omega^{c d}
$$

which is BRST invariant. Its anomalous dimension satisfies a Slavnov-Taylor identity and is simply related to the Faddeev-Popov ghost anomalous dimension, [29, 33.

To proceed to the effective potential that the operator satisfies we outline the local composite operator method, [30, 31, 32, in general terms. If we have an operator $\mathcal{O}$ then it is coupled to a source $J$ and the generating functional, $W[J]$, is constructed

$$
e^{-W[J]}=\int \mathcal{D} \Phi_{\mathrm{O}} \exp \left[-S+\int d^{d} x J_{\mathrm{O}} \mathcal{O}_{\mathrm{O}}+\frac{1}{2} \zeta_{\mathrm{o}} J_{\mathrm{O}}^{2}\right]
$$

where $\Phi$ represents the fields of the action $S$ and the subscript o corresponds to a bare quantity. After renormalization one has, [30, 31, 32],

$$
e^{-W[J]}=\int \mathcal{D} \Phi \exp \left[-S+Z_{\mathcal{O}} \int d^{d} x J \mathcal{O}+\frac{1}{2}(\zeta+\delta \zeta) J^{2}\right] .
$$

The quantity $\zeta$ is known as the LCO parameter and is a non-perturbative function of the coupling constant. It is defined to ensure that the renormalization group equation satisfied by $W[J]$ is homogeneous, [30, 31, 32]. As it has similar properties to a coupling constant it undergoes renormalization but we use the same notation for the counterterm, $\delta \zeta$, as [30, 31, 32]. The method to determine the explicit divergences contributing to $\delta \zeta$ was developed from the ideas of [30] in [40]. If one denotes the renormalization group function associated with the renormalization of $\zeta$ by $\delta(g)$ in the notation of [30] then $\zeta(g)$ is defined by the solution of

$$
\mu \frac{\partial \zeta}{\partial \mu}=2 \gamma_{\mathcal{O}}(g) \zeta+\delta(g)
$$

where $\gamma_{\mathcal{O}}(g)$ is the anomalous dimension of the operator whose effective potential we are interested in. Once $\zeta(g)$ is defined to the loop order required one evaluates $W[J]$ or applies a Hubbard-Stratonovich transformation to translate the source in the exponential within the path integral definition to a linear dependence, [30. This allows one to construct the effective potential using the standard procedures. Here the structure of $W[J]$ will be sufficient for our purposes as its dependence on $\mu_{\mathcal{I}}^{2}$ will determine the energetically favourable colour direction.

One main difference from the earlier application of the LCO formalism to $\frac{1}{2} A_{\mu}^{a} A^{a \mu}$ is that the operator (6) can be regarded as a sum of different operators which are colour projections of (8). In this respect one should have a vector of sources, $J_{\mathcal{I}}$, so that the operator source seed term in the initial application of the LCO formalism in (9) is

$$
\left[J_{\mathcal{Q}} \delta^{a c} \delta^{b d}+J_{\mathcal{W}} f^{a c e} f^{b d e}+\frac{J_{\mathcal{R}}}{C_{A}} f^{a b e} f^{c d e}+J_{\mathcal{S}} d_{A}^{a b c d}+\frac{J_{\mathcal{P}}}{N_{A}} \delta^{a b} \delta^{c d}+J_{\mathcal{T}} \delta^{a d} \delta^{b c}\right] \mathcal{O}^{a b c d} .
$$


From this we have performed the summation of one loop leg graphs for the $S U\left(N_{c}\right)$ colour group and found

$$
W[J]=-\frac{d N_{A} \gamma^{4} Z_{\gamma}^{4}}{2 g^{2} Z_{g}^{2}}+\frac{d N_{A} \zeta J \gamma^{2}}{g^{2}}+\frac{(d-1) N_{A}}{2} \int_{k} \ln \left[k^{2}\left[k^{2}+\frac{C_{A} \gamma^{4}}{\left[k^{2}+J\right]}\right]\right]+O\left(g^{2}\right)
$$

where $Z_{\gamma}$ and $Z_{g}$ are the respective renormalization constants for $\gamma$ and $g$ and

$$
J=\left[J_{\mathcal{Q}}+J_{\mathcal{R}}-J_{\mathcal{T}}+\frac{C_{A}}{2} J_{\mathcal{W}}\right]
$$

is the combination of sources which emerges from the formulation. The second term of (13) has no one loop divergences, [29]. We recall that to proceed to the effective potential one introduces a field $\sigma(x)$ which is the field which couples linearly to the source, 30, 31, 32], and in effect corresponds to the original composite operator. Consequently the effective potential for $\sigma$ emerges from the constant field value of the effective action for the operator given by

$$
\Gamma[\sigma]=W[J]-\int d^{4} x J(x) \sigma(x)
$$

after a Legendre transformation, [30, 31, 32. Hence, the one loop effective potential is

$$
\begin{aligned}
V\left(M^{2}\right)= & -\frac{2 N_{A} \gamma^{4}}{g^{2}}+\frac{4 N_{A} \zeta M^{2} \gamma^{2}}{g^{2}} \\
& +\frac{N_{A}}{64 \pi^{2}}\left[7 C_{A} \gamma^{4}+M^{4} \ln \left(\frac{M^{2}}{\mu^{2}}\right)+3 M^{2} \sqrt{\left[M^{4}-4 C_{A} \gamma^{4}\right]} \ln \left(\frac{a_{+}^{2}}{\mu^{2}}\right)\right. \\
& \left.-\frac{3}{4}\left[M^{4}-4 C_{A} \gamma^{4}\right] \ln \left(\frac{C_{A} \gamma^{4}}{\mu^{4}}\right)-\frac{3}{2} M^{2} \sqrt{\left[M^{4}-4 C_{A} \gamma^{4}\right]} \ln \left(\frac{C_{A} \gamma^{4}}{\mu^{4}}\right)\right]
\end{aligned}
$$

where $a_{+}^{2}=\frac{1}{2}\left[M^{2}+\sqrt{\left[M^{4}-4 C_{A} \gamma^{4}\right]}\right]$ and $\mu$ is the scale introduced to ensure that $g$ is dimensionless in $d$-dimensions. In the present context the effective potential depends on the unique combination of masses given by

$$
M^{2}=\left[\mu_{\mathcal{Q}}^{2}+\mu_{\mathcal{R}}^{2}-\mu_{\mathcal{T}}^{2}+\frac{C_{A}}{2} \mu_{\mathcal{W}}^{2}\right]
$$

where $M^{2}$ represents the constant field value of the corresponding $\sigma$ field. The potential has an absolute minimum at this value of $M^{2}$ as it is this specific combination which minimizes the potential. Any deviation away from this combination will increase it. However, considering the overall situation this means that not only does the original operator $\mathcal{O}^{a b c d}$ condense, it does so in a particular colour direction. To see what this is one computes the different combinations of $\mu_{\mathcal{I}}^{2}$ which emerge when the colour tensor

$$
\mathcal{T}^{a b c d}=\left[\mu_{\mathcal{Q}}^{2} \delta^{a c} \delta^{b d}+\mu_{\mathcal{W}}^{2} f^{a c e} f^{b d e}+\frac{\mu_{\mathcal{R}}^{2}}{C_{A}} f^{a b e} f^{c d e}+\mu_{\mathcal{S}}^{2} d_{A}^{a b c d}+\frac{\mu_{\mathcal{P}}^{2}}{N_{A}} \delta^{a b} \delta^{c d}+\mu_{\mathcal{T}}^{2} \delta^{a d} \delta^{b c}\right]
$$

is multiplied by each constituent tensor in turn. It is straightforward to see that, for $S U\left(N_{c}\right)$, $\mathcal{T}^{a b c d} f^{a b e} f^{c d e}=C_{A} M^{2}$. Thus at one loop from the LCO effective potential the general operator condensation is in the $\mathcal{R}$ colour direction since

$$
\left\langle\mathcal{O}^{a b c d}\right\rangle \propto f^{a b e} f^{c d e} .
$$

This preferred colour direction was suggested in [33] based on the structure of the propagators (5). Integrating over the localizing ghost propagators in the combination defining the BRST 
invariant dimension two operator, the only non-zero contribution comes from the massive propagator term in the $\xi_{\mu}^{a b}$ propagator. Note that this only applies above two dimensions as there are potential infrared divergences in strictly two dimensions. Indeed the zero momentum behaviour of the gluon in two dimensions is different from that in three and four dimensions. Lattice data, [41, 42, shows the gluon propagator is suppressed and vanishes at zero momentum unlike the non-zero freezing above two dimensions. In this respect the scaling solution appears to be preferred over the decoupling one. Aside from this caveat it is worth stressing that our result, (19), is derived in an explicit one loop analysis and the observation of [33] should be regarded as a trivial consistency check. Concerning three dimensions it is also the case that the $\mathcal{R}$ channel is the energetically favourable one. This can be seen from the summation of the graphs leading to $W[J]$ since the emergence of the combination (17) for the mass dependence is due to the underlying group theory. Integration over the loop momentum does not affect this. Instead it would lead to different values of the integral in (13) when the loop integration is performed. If one accepts that the natural solution is the $\mathcal{R}$ colour direction then the next stage is the observation that the operator $\mathcal{O}^{a b c d}$ condenses in this direction thereby giving non-zero masses to the localizing ghost fields. That one loop analysis was given in [33]. The localizing ghost propagator corrections have properties which distinguish them from the $\mathcal{Q}$ solution and could be tested on the lattice.

To conclude we have extended the analysis of [29] to consider the most general dimension two BRST invariant localizing ghost operator in the Gribov-Zwanziger Lagrangian of [33]. The computation indicates that at least to one loop the energetically favoured colour condensation channel is the $\mathcal{R}$ one. To proceed one would have to continue to the next loop order. This would be a huge task given the multiscale nature of the two loop massive vacuum bubble graphs which would arise. However, in the interim one hope would be that the infrared structure of the localizing ghost propagators could be analysed by other techniques. While the lattice could

provide numerical data the definition of an object on the lattice corresponding to say $\xi_{\mu}^{a b}$ is not straightforward. So a Schwinger-Dyson approach may offer the best avenue for an independent analysis.

Acknowledgements. This work was carried out in part with the support of an STFC studentship (DJT).

\section{References.}

[1] C. Feuchter \& H. Reinhardt, Phys. Rev. D70 (2004), 105021.

[2] A. Cucchieri \& T. Mendes, PoS LAT2007 (2007), 297.

[3] I.L. Bogolubsky, E.M. Ilgenfritz, M. Müller-Preussker \& A. Sternbeck, PoS LAT2007 (2007), 290.

[4] A. Maas, Phys. Rev. D75 (2007), 116004.

[5] A. Sternbeck, L. von Smekal, D.B. Leinweber \& A.G. Williams, PoS LAT2007 (2007), 304.

[6] I.L. Bogolubsky, E.M. Ilgenfritz, M. Müller-Preussker \& A. Sternbeck, Phys. Lett. B676 (2009), 69.

[7] A. Cucchieri \& T. Mendes, Phys. Rev. Lett. 100 (2008), 241601. 
[8] A. Cucchieri \& T. Mendes, Phys. Rev. D78 (2008), 094503.

[9] O. Oliveira \& P.J. Silva, Phys. Rev. D79 (2009), 031501.

[10] Ph. Boucaud, J.P. Leroy, A.L. Yaounac, J. Micheli, O. Pène \& J. Rodríguez-Quintero, JHEP 0806 (2008), 099.

[11] C.S. Fischer, A. Maas \& J.M. Pawlowski, Annals Phys. 324 (2009), 2408.

[12] V.N. Gribov, Nucl. Phys. B139 (1978), 1.

[13] F.J. Llanes-Estrada \& R. Williams, Phys. Rev. D86 (2012), 065034.

[14] A. Cucchieri \& T. Mendes, Phys. Rev. D88 (2013), 114501.

[15] D. Epple, H. Reinhardt \& W. Schleifenbaum, Phys. Rev. D75 (2007), 045011.

[16] H. Reinhardt, Phys. Rev. Lett. 101 (2008), 061602.

[17] G. Burgio, M. Quandt \& H. Reinhardt, Phys. Rev. Lett. 102 (2009), 032002.

[18] D. Zwanziger, Nucl. Phys. B209 (1982), 336.

[19] D. Zwanziger, Nucl. Phys. B321 (1989), 591.

[20] D. Zwanziger, Nucl. Phys. B323 (1989), 513.

[21] G. Dell'Antonio \& D. Zwanziger, Nucl. Phys. B326 (1989), 333.

[22] G. Dell'Antonio \& D. Zwanziger, Commun. Math. Phys. 138 (1991), 291.

[23] D. Zwanziger, Nucl. Phys. B364 (1991), 127.

[24] D. Zwanziger, Nucl. Phys. B378 (1992), 525.

[25] D. Zwanziger, Nucl. Phys. B399 (1993), 477.

[26] D. Zwanziger, Nucl. Phys. B412 (1994), 657.

[27] J.A. Gracey, Phys. Lett. B632 (2006), 282; Phys. Lett. B686 (2010), 319.

[28] D. Zwanziger, Phys. Rev. D81 (2010), 125027.

[29] D. Dudal, J.A. Gracey, S.P. Sorella, N. Vandersickel \& H. Verschelde, Phys. Rev. D78 (2008), 065047.

[30] H. Verschelde, K. Knecht, K. van Acoleyen \& M. Vanderkelen, Phys. Lett. B516 (2001), 307.

[31] H. Verschelde, Phys. Lett. B351 (1995), 242.

[32] H. Verschelde, S. Schelstraete \& M. Vanderkelen, Z. Phys. C76 (1997), 161.

[33] J.A. Gracey, Phys. Rev. D82 (2010), 085032.

[34] T. Kugo \& I. Ojima, Prog. Theor. Phys. Suppl. 66 (1979), 1; Prog. Theor. Phys. Suppl. 77 (1984), 1121.

[35] V. Mader, M. Schaden, D. Zwanziger \& R. Alkofer, arXiv:1309.0497. 
[36] J.A. Gracey, JHEP 0605 (2006), 052; JHEP 1002 (2010), 078.

[37] N. Maggiore \& M. Schaden, Phys. Rev. D50 (1994), 6616.

[38] D. Dudal, R.F. Sobreiro, S.P. Sorella \& H. Verschelde, Phys. Rev. D72 (2005), 014016.

[39] T. van Ritbergen, A.N. Schellekens \& J.A.M. Vermaseren, Int. J. Mod. Phys. A14 (1999), 41.

[40] R.E. Browne \& J.A. Gracey, JHEP 0311 (2003), 029.

[41] A. Maas, Phys. Rev. D75 (2007), 116004.

[42] A. Cucchieri \& T. Mendes, AIP Conf. Proc. 1343 (2011), 185. 